\title{
Factores ambientales relacionados con la cobertura de Agave angustifolia (Asparagaceae) en el matorral costero de Yucatán, México
}

\section{Environmental factors associated with the cover of Agave angustifolia (Asparagaceae) in the coastal scrubland of Yucatán, Mexico}

\author{
José Carlos Cervera Herrera' (D), Jorge L. Leirana-Alcocer',2 (D), Jorge A. Navarro Alberto' (D)
}

1 Universidad Autónoma de Yucatán, Cuerpo Académico de Ecología Tropical, Campus de Ciencias Biológicas y Agropecuarias, Yucatán, México.

2 Autor para la correspondencia: jleirana@correo.uady.mx

Recibido: 19 de mayo de 2017. Revisado: 18 de octubre de 2017

Aceptado: 11 de enero de 2018.

Primero en línea: 29 de mayo de 2018.

Publicado: 1 de julio de 2018.

\section{Citar como:}

Cervera Herrera, J. C., J. L. LeiranaAlcocer y J. A. Navarro Alberto. 2018. Factores ambientales relacionados con la cobertura de Agave angustifolia (Asparagaceae) en el matorral costero de Yucatán, México. Acta Botanica Mexicana 124: 75-84. DOI: 10.21829/ abml24.2018.1252

DOI:

10.21829/abml24.2018.1252

\section{Resumen:}

Antecedentes y Objetivos: Agave angustifolia, o chelem, es el único maguey silvestre de la Península de Yucatán y es el ancestro de especies domesticadas de gran valor económico y cultural. Presta importantes servicios ambientales como recurso alimenticio para la fauna, fijación de suelos arenosos y captura de carbono en ambientes áridos con suelos pobres. Es un cultivo potencial de cara al aumento de las sequías predichas para la región y del empobrecimiento genético del henequén. El objetivo de este trabajo es recabar datos sobre la densidad de sus poblaciones, su patrón espacial, estructura poblacional y la relación entre su abundancia y los factores ambientales en el matorral costero de Yucatán, con el fin de evaluar su uso en la restauración ecológica.

Métodos: Se muestrearon tres localidades costeras: Chuburná (Ch), San Benito (SB) y Río Lagartos (RL). En SB y RL se trazaron 36 cuadrantes de $4 \mathrm{~m}^{2}$; dentro de cada uno se estimó el porcentaje de cobertura de Agave angustifolia, el de los arbustos de más de $1 \mathrm{~m}$ de alto y la cobertura de mantillo. Se tomaron muestras de suelo a las que se analizó pH, conductividad eléctrica, nitrógeno total, materia orgánica y escala de gris. Se describieron la densidad poblacional, distribución espacial y estructura por tamaños de $A$. angustifolia en SB y Ch.

Resultados clave: La cobertura de Agave angustifolia se correlaciona negativamente con la cobertura de arbustos y mantillo, y cantidad de materia orgánica. Las densidades de su población fueron inferiores a 0.1 individuos $/ \mathrm{m}^{2}$, hubo una mayor proporción de plántulas y su distribución espacial es agregada.

Conclusiones: Al soportar suelos pobres, altas temperaturas y radiación solar, la especie puede servir para restaurar algunas funciones ecológicas en las zonas más áridas de la costa donde la vegetación ha sido severamente dañada.

Palabras clave: Arenosol, distribución espacial, estructura poblacional, suculenta, taxon emparentado con cultivos, zonas semiáridas.

\section{ABSTRACT:}

Background and Aims: Agave angustifolia, or chelem, is the only wild species of the Yucatán Peninsula and is the ancestor of cultivated species with great economic and cultural values. It provides important environmental services such as food source for fauna, it fixes sandy soils and sequesters carbon in arid habitats with poor soils. It is a potential crop that may face the dryness exacerbation expected for the region and the genetic erosion of henequen. The aim of this work is to gather data about its population density, spatial pattern, size structure, and the relationship between its abundance and environmental factors of the Yucatán coastal scrublands, in order to assess the use of the species for ecological restauration purposes.

Methods: Field work was carried out in three coastal sites: Chuburná (Ch), San Benito, (SB) and Río Lagartos (RL). Thirty six $4 \mathrm{~m}^{2}$ plots were placed in SB and RL; we estimated the relative cover of Agave angustifolia, shrubs higher than $1 \mathrm{~m}$ and litter in each plot. Potential of Hidrogen $(\mathrm{pH})$, electric conductivity, total nitrogen, organic matter and gray scale were measured from the soil samples collected. Population density, spatial pattern and size structure of $A$. angustifolia in SB and Ch were also described. Key results: The cover of Agave angustifolia is negatively correlated to the shrubs and litter cover, as well as the amount of organic matter. The population density was lower than 0.1 individuals $/ \mathrm{m}^{2}$, small individuals dominated the population structure and the spatial pattern is aggregated.

Conclusions: Because its ability to live in poor soils, high temperatures and solar radiation, the species could be used to restore some ecological functions in the most arid habitats of the coast where the vegetation has been severily damaged.

Key words: Arenosol, crop related taxa, population structure, semiarid zones, spatial distribution, succulent. 


\section{INTRODUCCIÓN}

Agave angustifolia Haw., o chelem, como es llamado en lengua maya, es el único maguey silvestre de la Península de Yucatán y es el ancestro más probable de especies domesticadas de gran valor económico y cultural como el henequén, el sisal y el agave azul (Colunga-García Marín et al., 1996). Es un recurso de importancia comercial en México y otras partes del mundo pues proporciona la materia prima de diferentes productos industrializados como fibra, jarabe edulcorante, biocombustible y bebidas alcohólicas (García-Mendoza et al., 1993; Salazar-Solano y Mungaray-Lagarda, 2009).

Esta especie puede, así mismo, ser un cultivo alternativo que proporcione cierta resiliencia a los sistemas rurales de producción, de cara al aumento de las sequías que se pronostican para la Península de Yucatán (HernándezCerda y Valdez-Madero, 2004) y al empobrecimiento genético del henequén (Colunga-García Marín et al., 1996).

En los ecosistemas en los que esta especie está presente, sus poblaciones naturales prestan importantes servicios ambientales, ya que sus flores y frutos proporcionan alimento a quirópteros, aves e insectos nectarívoros y granívoros, sus raíces fijan el suelo en dunas arenosas y sus individuos secuestran carbono en ambientes áridos con suelos pobres (Rocha et al., 2006; García-Moya et al., 2011; Medina-van Berkum et al., 2016).

Agave angustifolia es un buen candidato para ser reintroducido a ecosistemas degradados, ya que es resistente a las condiciones estresantes como la escasez de agua y altas temperaturas (García-Moya et al., 2011). Sin embargo, para conservarlo es necesario recabar información sobre los factores ambientales asociados a su cobertura relativa y conocer la densidad y estructura de sus poblaciones.

En el caso de los agaves y otras suculentas, entre los factores más importantes que determinan su cobertura relativa se encuentran el acceso a la luz y la competencia por ella con arbustos o árboles; en estudios experimentales también se ha documentado la importancia de algunas propiedades del suelo como el contenido de materia orgánica y el nitrógeno (Nobel, 1988).
En especies longevas, la estructura por tamaños o etapas de vida de una población, es el resultado de los procesos de reclutamiento y mortalidad pasados; conocerlos nos da una idea de la historia de la especie en una localidad. De igual manera, la estructura determina en gran medida su futuro a corto plazo, ya que incide en las tasas de crecimiento poblacional (Krebs, 2009).

La densidad real de las especies nos proporciona un indicador de los sitios en los que la especie es más abundante o escasa, pero sin tomar en cuenta el arreglo espacial de la población dentro del hábitat y de qué manera ocupa los diferentes microambientes que existen en él. La densidad ecológica, por otro lado, proporciona información sobre los mecanismos de dispersión de la especie, de las interacciones entre sus individuos y de su distribución en los diferentes microhábitats.

En las especies de crecimiento clonal, sobre todo en fragmentos con condiciones favorables, los propágulos se producen en abundancia y muy cerca de la planta madre; estos clones tienden a tener una alta sobrevivencia, por lo que las densidades ecológicas tienden a ser más altas que cuando la propagación principal es mediante semillas, o en microambientes menos propicios.

El objetivo de este trabajo es recabar datos sobre la relación entre la cobertura relativa de A. angustifolia y algunos factores ambientales, la densidad de las poblaciones, su patrón espacial y estructura poblacional en el matorral costero de Yucatán, lo que determinará su potencial en la restauración ecológica de este ecosistema.

\section{Materiales y Métodos}

\section{Área de estudio}

El estudio se llevó a cabo en las siguientes tres localidades de la costa de Yucatán (Fig. 1): Chuburná (Ch) (21 ${ }^{\circ} 15^{\prime} 44.51 " \mathrm{~N}$, $\left.89^{\circ} 50^{\prime} 13.5^{\prime \prime O}\right)$, San Benito (SB) $\left(21^{\circ} 19^{\prime} 44.51^{\prime \prime} \mathrm{N}\right.$, $\left.89^{\circ} 24^{\prime} 08.8^{\prime \prime O}\right)$ y Río Lagartos (RL) $\left(21^{\circ} 36^{\prime} 40.71 " \mathrm{~N}\right.$, $88^{\circ} 03^{\prime} 10.3^{\prime \prime O}$ ), en el paisaje geomorfológico de "cordón litoral" en el que los suelos son arenosoles calcáreos de colores muy claros y de origen marino (Bautista et al., 2015). Este suelo presenta buen drenaje y no es capaz de almacenar 
el agua y los nutrimentos de manera eficaz (León-Arteta, 2003). El contenido de materia orgánica, nitrógeno y otros elementos se correlaciona con la cobertura de los arbustos (Leirana-Alcocer y Bautista, 2014), de manera que cuando la vegetación es destruida, el suelo es incapaz de retener estos nutrimentos por mucho tiempo. El clima en las áreas de estudio oscila entre el cálido seco (BS), con precipitaciones anuales menores a los $600 \mathrm{~mm}$ y el cálido subhúmedo (AW) con precipitaciones de $800 \mathrm{~mm}$. Las temperaturas medias anuales son superiores a $\operatorname{los} 25^{\circ} \mathrm{C}$ en toda la costa.

La vegetación en este paisaje se denomina matorral de duna costera o matorral costero (Flores y Espejel, 1994; Espejel et al., 2017), con características fisionómicas de un matorral xerófilo tales como la dominancia de arbustos espinosos, esclerófilos y abundancia de especies suculentas como las cactáceas y los agaves (González-Medrano, 2004). Algunas de las especies dominantes en el estrato arbustivo del matorral costero son Coccoloba uvifera (L.) L., Pithecellobium keyense Britton, Caesalpinia vesicaria L., Metopium brownei (Jacq.) Urb. y Bumelia retusa Sw., los cuales pueden servir de hospedero a bromelias epífitas como Tillandsia sp. u orquídeas como Myrmecophila christinae Carnevali \& Gómez-Juárez. También son fre- cuentes algunas cactáceas como Opuntia stricta (Haw.) Haw., Acanthocereus tetragonus (L.) Hummelinck, Selenicereus donkelaarii (Salm-Dyck) Britton \& Rose y Mammillaria gaumeri (Britton \& Rose) Orcutt. Los agaves pueden ser espacialmente raros, aunque existen fragmentos en los que su densidad es muy alta ( $>5$ individuos $/ \mathrm{m}^{2}$ ).

\section{Relación Agave angustifolia-ambiente}

Para determinar la correlación entre la cobertura de Agave angustifolia y algunas propiedades del suelo y la vegetación se usaron datos colectados en abril de 2008 en San Benito (SB) y en Río Lagartos (RL). En cada sitio se delimitaron dos transectos de $180 \mathrm{~m}$ de largo perpendiculares a la línea de costa. Sobre cada transecto se marcaron 18 cuadrantes de $4 \mathrm{~m}^{2}$ cada 10 metros. Dentro de cada uno de estos cuadrantes se midió el porcentaje de cobertura de mantillo (MAN), el de arbustos de más de $1 \mathrm{~m}$ de altura (ARB) y el de $A$. angustifolia (AGV).

En cada cuadrante se tomó una muestra de $100 \mathrm{~cm}^{3}$ de los primeros $5 \mathrm{~cm}$ de suelo, utilizando cilindros de $\mathrm{PVC}$ de $5.08 \mathrm{~cm}$ de diámetro y $5 \mathrm{~cm}$ de largo; no se incluyó el mantillo superficial en la muestra. Las muestras de suelos se secaron a temperatura ambiente hasta peso constante,

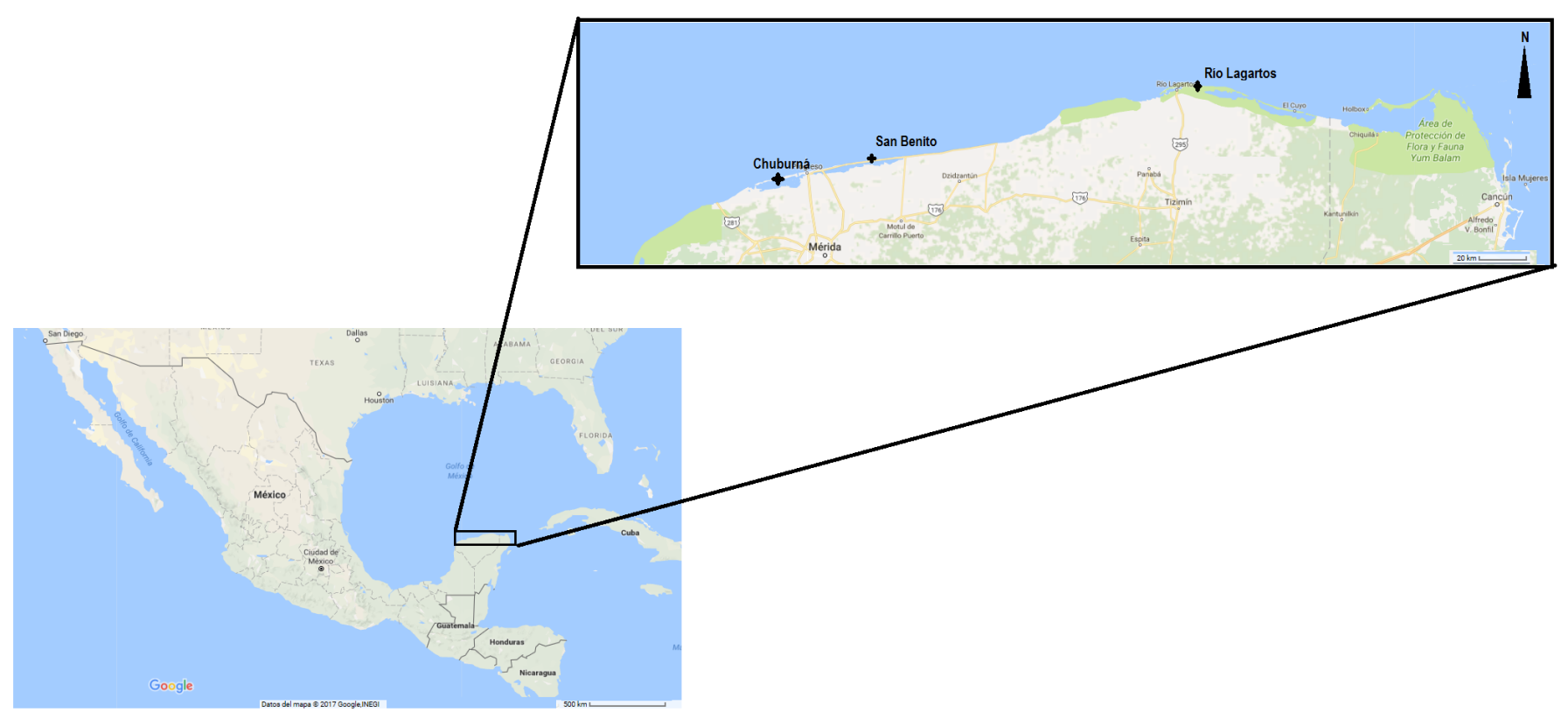

Figura 1: Localización de los sitios de estudio (Chuburná, San Benito y Río Lagartos) en la costa de Yucatán, México. 
se midió densidad aparente (relación peso-volumen en g/ $\mathrm{cm}^{3}$ ), porcentaje de materia orgánica (oxidación húmeda), contenido de nitrógeno total en $\mathrm{mg} / \mathrm{kg}$ (destilación), $\mathrm{pH}$ de solución 1:10 por el método de potenciómetro (medidor de electrodo de gel modelo HI 9124, Hannah Instruments, Woonsocket, RI, EUA) y conductividad eléctrica en $\mu \mathrm{S} / \mathrm{cm}$ (conductímetro modelo HI 9033, Hannah Instruments, Woonsocket, RI, EUA) en una solución acuosa 1:10. Por último, se comparó el color de las muestras de suelo con la carta de escala de grises Q13 de Kodak; a cada muestra de suelo se le asignó un valor de escala de gris según su semejanza con el patrón correspondiente de la carta (Leirana-Alcocer y Bautista, 2014).

Se realizaron análisis de correlación simple entre estas variables y la cobertura de A. angustifolia usando el programa Statgraphics Centurion XV plus (StatPoint Inc., 2005). Adicionalmente, se llevó a cabo un análisis de correlación parcial entre las variables ARB, MAN y oscuridad, para dilucidar cuál de ellas explicaba mejor la variación en la cobertura de $A$. angustifolia, ya que todas ellas se correlacionaban fuertemente entre sí. Se reportan primero las correlaciones simples en forma de cuadro y enseguida los resultados de las correlaciones parciales.

\section{Estructura poblacional y patrón espacial}

Para describir la estructura poblacional y el patrón espacial de Agave angustifolia, en noviembre de 2016 realizamos búsquedas intensivas de individuos en Chuburná y San Benito, dos de los sitios de estudio. En cada sitio se trazaron dos cuadrantes rectangulares de $800 \mathrm{~m}^{2}$ cada uno, sus lados más largos eran perpendiculares a la línea costera y medían $200 \times 4 \mathrm{~m}$. Los transectos solamente incluyeron la vegetación de matorral costero que se desarrolla a una distancia de 40 a $50 \mathrm{~m}$ de la playa. A los individuos así encontrados se les midió su altura máxima (excluyendo la inflorescencia), longitud de la hoja más larga y diámetro de la copa. Además, se midió la distancia de cada individuo al conespecífico más cercano, a este valor se le llamará en lo sucesivo "distancia mínima".

Las variables indicadoras de tamaño (altura, longitud de la hoja y número de hojas) presentaron una muy alta correlación. Por lo tanto, se optó por usar el diámetro de la copa de los individuos para clasificarlos en clases de tamaños, ya que este valor será más fácil de comparar con otros estudios publicados. Los individuos se dividieron en seis clases de tamaño según el diámetro de su copa, siguiendo a Arias-Medellín et al. (2016): I) 0-5 cm, II) $5.1-20 \mathrm{~cm}$, III) $20.1-50 \mathrm{~cm}$, IV) $50.1-100 \mathrm{~cm}$, V) 100.1 $150 \mathrm{~cm}$ y VI) $>150 \mathrm{~cm}$. Se registraron los individuos que estaban produciendo inflorescencias para recabar los datos sobre los tamaños de los sexualmente reproductivos.

Se estimó la densidad real al dividir el número de individuos encontrados en cada sitio entre la superficie muestreada, que fue de $1600 \mathrm{~m}^{2}$ en ambos casos.

La distancia mínima sirvió para estimar tanto la densidad ecológica como el índice de agregación, también se probó la hipótesis de que este parámetro variaba entre individuos de diferente tamaño.

La densidad ecológica se define como la densidad de individuos en la fracción del hábitat usada por la especie y se estimó usando la siguiente fórmula (Krebs, 1998):

$$
\widehat{N}=n_{/ \pi \sum} r_{i}^{2}
$$

En donde: $\widehat{N}$ es la densidad ecológica estimada, $n$ es el número de individuos muestreados y $r_{i}$ es la distancia entre el individuo $i$ y su conespecífico más cercano.

El índice de agregación espacial que estimamos fue el de Clark y Evans, (1954, citado en Krebs, 1998) con su respectiva probabilidad $\mathrm{P}$.

Para probar si las distancias mínimas variaban con la clase de tamaño se realizó un análisis de varianza en el que la distancia mínima fue transformada a $\ln (\mathrm{x})$ para cumplir los supuestos de normalidad de residuos y homogeneidad de varianza; se comprobó que sí se cumplieron.

\section{Resultados}

\section{Relación Agave angustifolia-ambiente}

En el cuadro 1 se presentan las correlaciones simples de las siguientes variables: cobertura relativa de $A$. angustifolia, cobertura de arbustos $>1 \mathrm{~m}$ de alto, cobertura de 
Cuadro 1: Correlaciones entre las variables ambientales medidas y la cobertura de Agave angustifolia Haw. en las localidades de San Benito (SB) y Río Lagartos (RL), Yucatán, México. Cobertura de arbustos $>1 \mathrm{~m}$ de alto (ARB), cobertura de mantillo (MAN), conductividad eléctrica del suelo (CE), escala de gris (EG), porcentaje de materia orgánica del suelo (MO \%), densidad aparente (DA), nitrógeno total (NT), diferencias no significativas (NS).

\begin{tabular}{|c|c|c|c|c|c|c|c|c|}
\hline & Cobertura Agave angustifolia Haw. & MAN & DA & NT & MO \% & $\mathrm{pH}$ & $\mathrm{CE}$ & EG \\
\hline ARB & $-0.37(0.04)$ & $0.60(<0.01)$ & NS & $0.39(0.04)$ & NS & NS & $0.56(0.023)$ & NS \\
\hline MAN & $-0.45(0.02)$ & & NS & $0.55(0.01)$ & NS & NS & NS & NS \\
\hline DA & NS & & & $-0.44(0.02)$ & $-0.75(<0.01)$ & NS & NS & $0.80(<0.01)$ \\
\hline NT & NS & & & & $0.87(<0.01)$ & NS & NS & $-0.52(<0.01)$ \\
\hline MO \% & NS & & & & & NS & NS & $-0.81(<0.01)$ \\
\hline $\mathrm{pH}$ & NS & & & & & & $0.6(<0.01)$ & NS \\
\hline $\mathrm{CE}$ & NS & & & & & & & $-0.62(0.01)$ \\
\hline EG & $-0.39(0.03)$ & & & & & & & \\
\hline
\end{tabular}

mantillo, conductividad eléctrica del suelo, escala de gris del suelo, materia orgánica del suelo, densidad aparente y nitrógeno total del suelo en San Benito (SB) y Río Lagartos (RL). En el cuadro 2 se presentan los valores promedio y las desviaciones estándar de las variables edáficas de ambas localidades.

La cobertura de arbustos de más de $1 \mathrm{~m}$ de alto (ARB) y las variables asociadas a la fertilidad del suelo, oscuridad y mantillo (MAN) se correlacionaron negativa-

Cuadro 2: Medias y desviaciones estándar (entre paréntesis) de las variables edáficas por sitio (San Benito y Río Lagartos). Cobertura de arbustos $>1 \mathrm{~m}$ de alto (ARB), cobertura de mantillo (MAN), conductividad eléctrica del suelo $(\mathrm{CE})$, escala de gris $(\mathrm{EG})$, porcentaje de materia orgánica del suelo (MO \%), densidad aparente (DA), nitrógeno total (NT).

\begin{tabular}{ccc}
\hline Variable & San Benito & Río Lagartos \\
\hline ARB & $76(50)$ & $86(52)$ \\
MAN & $70(33)$ & $72(26)$ \\
DA & $1.2(0.07)$ & $1.1(0.11)$ \\
NT & $0.14(0.05)$ & $0.2(0.11)$ \\
$\mathrm{MO} \%$ & $0.89(0.80)$ & $2.6(2.7)$ \\
pH & $7.3(0.21)$ & $7.4(0.5)$ \\
$\mathrm{CE}$ & $1042(664)$ & $1152(990)$ \\
$\mathrm{EG}$ & $13(7.4)$ & $14.8(6.6)$ \\
\hline
\end{tabular}

mente con la cobertura de $A$. angustifolia. Las otras variables no presentaron correlaciones significativas.

En las correlaciones parciales de estas tres variables con A. angustifolia se encontró que las más altas fueron con MAN cuando se controlaban tanto el efecto de oscuridad $(\mathrm{r}=-0.59, \mathrm{P}<0.01)$ como de ARB $(\mathrm{r}=-0.47$, $\mathrm{P}=0.01$ ). Cuando se controlaba el efecto de MAN ninguna resultó significativa. Esto indica que la variable independiente más asociada con $\mathrm{A}$. angustifolia es la cobertura de mantillo.

\section{Estructura poblacional y patrón espacial}

En San Benito la categoría de tamaño con mayor frecuencia fue la II (de 5.1 a $20 \mathrm{~cm}$ de diámetro de la copa), mientras que en Chuburná fue la III $(20.1 \mathrm{a} 50 \mathrm{~cm})$. En ambos sitios la categoría I $(<5 \mathrm{~cm})$ tuvo muy baja frecuencia relativa. En Chuburná no se encontraron individuos de la categoría VI, que son individuos mayores a $150 \mathrm{~cm}$ (Fig. 2).

El tamaño promedio de las plantas que presentaron inflorescencia fue de $120 \mathrm{~cm}$ de altura (con valores extremos entre 75 y $200 \mathrm{~cm}$ ), $75 \mathrm{~cm}$ de longitud de hoja (extremos 50 y $115 \mathrm{~cm}$ ) y una copa de $153 \mathrm{~cm}$ de diámetro (extremos 70 y $300 \mathrm{~cm}$ ). En Chuburná, más de 50\% de los individuos en este intervalo de tamaño presentaron inflorescencia, en tanto que en San Benito solamente 26\% (noviembre de 2016). 


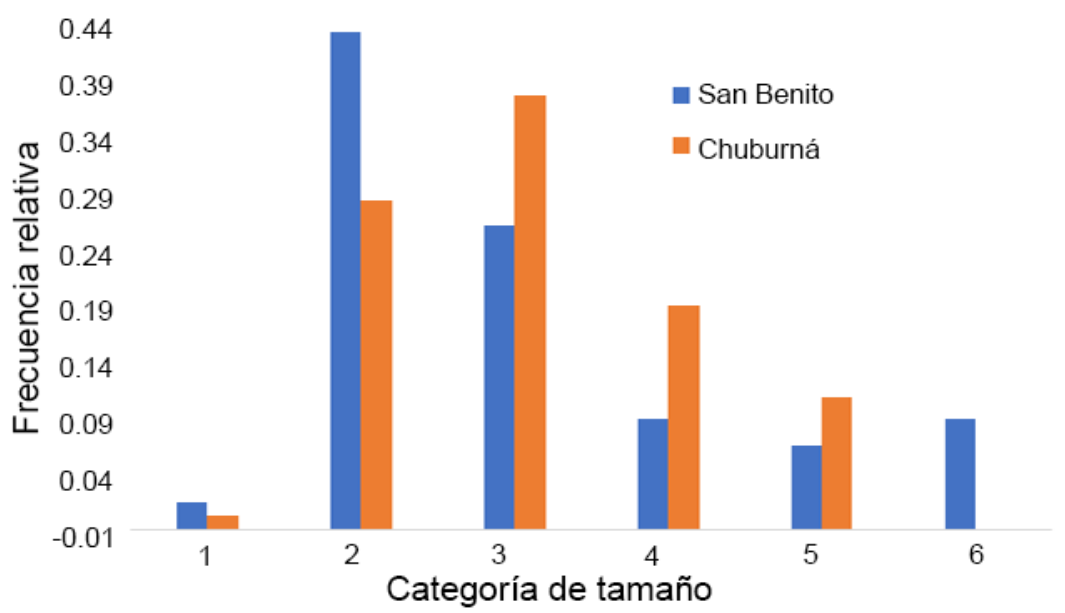

Figura 2: Estructura por tamaños de las poblaciones de Agave angustifolia Haw. en Chuburná (n=80) y San Benito ( $\mathrm{n}=128)$, dos localidades de la costa de Yucatán. Clases de diámetro de la copa en cm: I) 0-5, II) 5.1-20, III) 20.1-50, IV) 50.1-100, V) 100.1-150, VI) >150.

La densidad real en las parcelas muestreadas fue de 0.09 y 0.05 individuos por metro cuadrado en San Benito y Chuburná, respectivamente. La densidad ecológica, es decir la densidad de la especie dentro de la fracción del hábitat ocupado (estimado mediante las distancias mínimas) fue en San Benito de 0.42 y 0.36 individuos $/ \mathrm{m}^{2}$ en Chuburná. En ambas poblaciones los individuos se distribuyen de manera agregada (índice de agregación 0.3 en Chuburná y 0.2 en San Benito, $\mathrm{P}<0.01$ en ambos casos).

Las distancias mínimas fueron diferentes en individuos de diferente clase de tamaño, como se muestra en los resultados de la prueba de ANOVA (Cuadro 3). En la figura 3 se muestra la gráfica de medias y error estándar; los literales iguales indican grupos sin diferencias significativas. La Prueba Múltiple de Rangos indicó que la distancia mínima en plántulas (clases I y II de tamaño) era menor que en casi todos los demás grupos. Se observó una tendencia a tener una mayor densidad ecológica de individuos pequeños que mayores.

\section{DISCUSIÓN}

Los resultados sugieren que la cobertura de otros arbustos que compiten por luz y el mantillo que cubre el suelo, son factores que están fuertemente asociados a la dismi- nución de la dominancia relativa de $A$. angustifolia. Esto concuerda con los datos experimentales de este y otros agaves que demuestran que la radiación fotosintéticamente activa, junto con el agua, es uno de los principales factores limitantes de las tasas fotosintéticas en individuos juveniles y adultos (Winter et al., 2014).

En Chuburná se ha documentado que la densidad del flujo de fotones fotosintéticamente activos disminuye drásticamente (hasta $20 \%$ del flujo ambiental) bajo el follaje de arbustos muy densos (Hernández-Mendoza y colaboradores, datos sin publicar), lo que ocasiona que la cobertura de herbáceas sea muy pequeña. Los sitios en los que la cobertura del mantillo es mayor, y el suelo contiene más materia orgánica (y tiende a presentar una pigmentación más oscura), probablemente son aquellos en los que el matorral tiene más tiempo de haberse establecido. Por lo tanto, no solamente las condiciones actuales son adversas, sino que el fragmento tiene una historia en la que las condiciones han sido subóptimas para el crecimiento y sobrevivencia de A. angustifolia. Sin embargo, no se puede descartar que el mantillo y el color del suelo pueden también tener un efecto directo sobre las semillas y propágulos de agave, al modificar la calidad de la luz que llega al suelo. 
Cuadro 3: Anova de las diferencias en las distancias mínimas de individuos de Agave angustifolia Haw. de diferentes clases de tamaños en Chuburná y San Benito, Yucatán.

\begin{tabular}{lccccc}
\hline Fuente & Suma de cuadrados & GL & Cuadrados medios & Razón F & P \\
\hline Entre clases de tamaño & 3.58 & 5 & 0.72 & 7.99 & $<0.001$ \\
Dentro de clases de tamaño & 10.67 & 119 & 0.09 & & \\
Total & 14.26 & 124 & & & \\
\hline
\end{tabular}

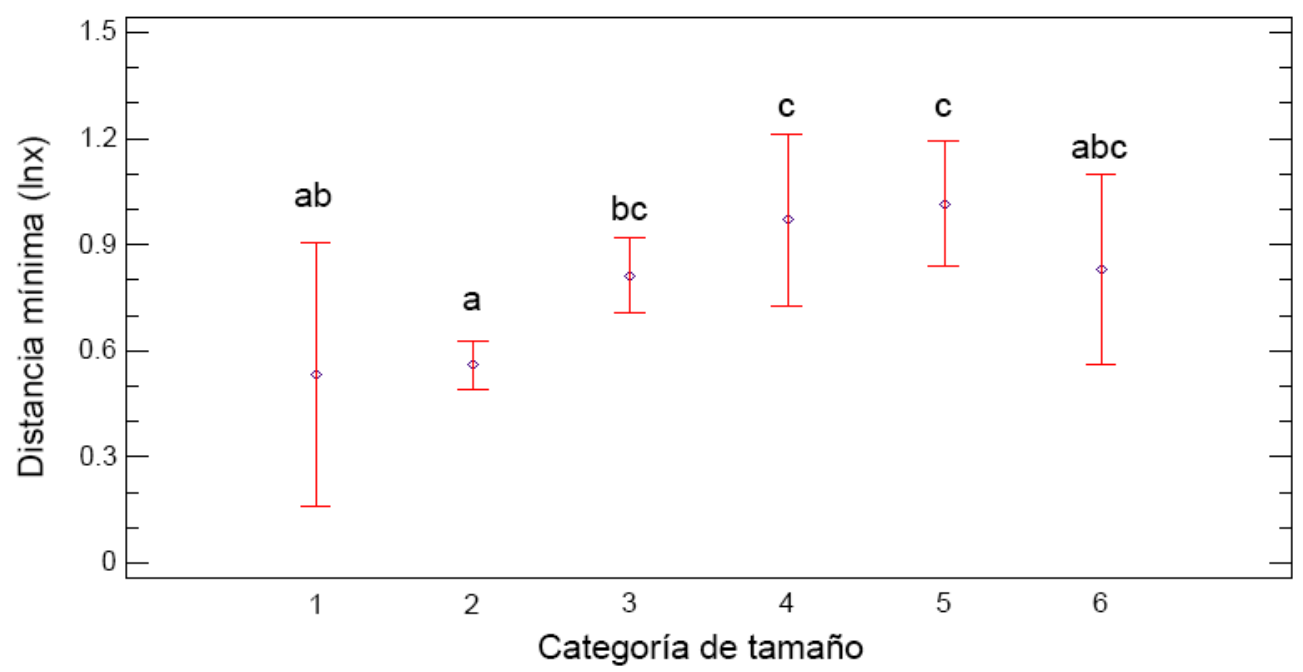

Figura 3: Promedio y error estándar de las distancias mínimas en metros $(\ln (\mathrm{x}))$ entre individuos de Agave angustifolia Haw. por clase de tamaño en Chuburná y San Benito, Yucatán. Clase de tamaño según el diámetro de la copa en cm: I) 0-5, II) 5.1-20, III) 20.1-50, IV) 50.1-100, V) 100.1$150, \mathrm{VI})>150$.

La cubierta de mantillo, al filtrar la luz, hace que disminuya la razón: rojo/rojo lejano de la radiación que llega hasta el suelo (Vázquez-Yañes et al., 1990), lo que puede inhibir la germinación de semillas y la producción de propágulos de algunas especies de crecimiento clonal (Oborny, 1994). En este tipo de vegetación, en los fragmentos con matorrales densos, se puede formar una capa de hojarasca de más $2 \mathrm{~cm}$ de espesor (obs. pers.), que se acumula debido a su lenta descomposición producida por baja humedad característica de los climas semiáridos.

En la literatura se reporta que la tasa de crecimiento de $A$. angustifolia y otras especies del mismo género se correlaciona positivamente con algunas variables que determinan la calidad del suelo cuando no existen otros factores limitantes (Nobel, 1988; García-Moya et al.,
2011). De hecho, cuando se controla el efecto de los otros factores, solamente la cobertura de mantillo tiene una asociación fuerte con la cobertura de A. angustifolia. Es conocida la capacidad de los agaves de sobrevivir en suelos pobres y secos (Ramírez-Tobías et al., 2014; Owen et al., 2015), y su habilidad de asociarse con hongos micorrizógenos (Carmona-Escalante et al., 2013), que le permiten aumentar su eficiencia en la adquisición de agua y nutrimentos edáficos.

La estructura por tamaños refleja el hecho de que la germinación o sobrevivencia de plántulas es muy baja, como es característico en plantas de vida larga en ecosistemas áridos y semiáridos (Nobel, 1988). La alta frecuencia relativa de individuos de tamaño II y III puede ser consecuencia de que cada individuo es capaz de producir varios 
clones en estas categorías, sobre todos los originados por rizomas. Se ha reportado que estos clones tienen una alta tasa de sobrevivencia gracias al aporte de agua y fotosintatos de la planta madre (Badano y Pugnaire, 2004). En este estudio se observó que individuos menores a $50 \mathrm{~cm}$ de altura ya son capaces de producir clones rizomatosos.

Las poblaciones de $A$. angustifolia podrían mantenerse, e incluso aumentar, debido a que en ambos sitios hay perturbaciones de la vegetación como la tala y poda de vegetación arbustiva, lo que incrementa la cantidad de luz que penetra hasta el suelo. No obstante, la extracción y destrucción de individuos adultos podría revertir este efecto, ya que en el género Agave L. el reclutamiento de nuevos adultos a partir de semillas es poco frecuente (Jordan y Nobel, 1979; Nobel, 1988).

La densidad real de $A$. angustifolia es muy baja porque la vegetación en los sitios de estudio consiste en un matorral muy cerrado, con arbustos que generan una sombra muy espesa, que como ya se vio está asociada negativamente a la cobertura de agave. La densidad ecológica, la cual indica la densidad en la fracción de hábitat realmente ocupada por la especie, es entre cuatro y siete veces mayor que su densidad real. Esto es posible porque la distribución espacial, tanto de la especie como de sus microambientes favorables, resultó ser fuertemente agregada. Las especies de crecimiento clonal tienden a producir más clones por unidad de área en microambientes favorables (Oborny, 1994). Esto les permite reducir la probabilidad de que un organismo competidor se establezca y los desplace y compensa por la baja productividad en microambientes desfavorables del hábitat.

Asimismo, aunque la reproducción por semillas podría generar un patrón menos agregado, la germinación y sobrevivencia para magueyes silvestres en ambientes naturales es un evento muy poco frecuente (menor a 1\%) (Jordan y Nobel, 1979; Ramírez-Tobías et al., 2014), como se pudo notar en la menor frecuencia relativa de individuos de clase de tamaño I. Es probable que la reproducción sexual sea importante en el mantenimiento de la diversidad y en la generación de nuevas combinaciones genéticas; sin embargo, se ha reportado que su contribu- ción demográfica es prácticamente nula y el mantenimiento y crecimiento de las poblaciones ocurre principalmente por reproducción asexual (Arizaga y Ezcurra, 2002).

La población tuvo una distribución agregada y hubo una tendencia a que los individuos más pequeños tuvieran distancias menores a su vecino más cercano, quizá debido a que las plántulas sufren menos por la competencia de sus congéneres, pero ésta aumenta de intensidad a medida que las plantas aumentan su cobertura y masa radicular.

Aunque la sombra parcial reduce la tasa fotosintética de agaves adultos y juveniles, en otras especies del género, las plántulas originadas a partir de semillas requieren de sombra parcial para poder sobrevivir y crecer en ambientes cálidos y secos (Jordan y Nobel, 1979; Franco y Nobel, 1988). En el caso de Agave angustifolia, para individuos más pequeños, las tasas de fotosíntesis y crecimiento son mayores en individuos que reciben $50 \%$ de la luz ambiental total que las iluminadas con mayor o menor intensidad (Cervera, datos sin publicar).

En este sentido, la reproducción clonal, presente en agaves, cactáceas y otras especies xerófitas es una clara ventaja, pues los propágulos presentan una baja tasa de mortalidad, aún en condiciones de alta radiación, al recibir agua y fotosintatos de la planta madre (Badano y Puignare, 2004; García-Moya et al., 2011).

La densidad real fue mayor en San Benito, probablemente porque en ese lugar se da "limpieza" frecuente a los terrenos, la cual consiste en la tala y poda de arbustos, lo que provoca un aumento de la tasa de crecimiento y cobertura de A. angustifolia, como sugieren las correlaciones negativas entre cobertura de arbustos y de agave.

\section{CONCLUSIONES}

Agave angustifolia es una especie que, al resistir condiciones de alta radiación y escasez de nutrimentos del suelo, tiene potencial para restaurar algunas funciones ecosistémicas del matorral costero de Yucatán, en especial áreas en las que se ha destruido la cobertura arbustiva. Los datos sugieren que la abundancia de la especie puede ser alta en ambientes en los que la cobertura arbustiva y de mantillo es escasa, ya que las condiciones de alta radia- 
ción favorecen el crecimiento individual y probablemente la producción de clones en el rizoma.

En las poblaciones de Chuburná y San Benito ha habido poco reclutamiento de plántulas menores a $5 \mathrm{~cm}$ de diámetro de copa, lo que es común para especies longevas en ambientes semiáridos y cálidos (Franco y Nobel, 1988; Arias-Medellín et al., 2016; Zepeda et al., 2017).

\section{CONTRIBUCIÓN DE AUTORES}

JL y JC concibieron y diseñaron el estudio. JN y JL realizaron los análisis estadísticos. Todos los autores contribuyeron a la adquisición de datos y su interpretación. JL escribió el manuscrito con la ayuda de los otros autores. Todos los autores contribuyeron a la discusión, revisión y aprobación del manuscrito final.

\section{FINANCIAMIENTO}

Este trabajo fue apoyado por el Programa para el Desarrollo Profesional Docente (PRODEP) de la Secretaría de Educación Pública, mediante el proyecto "Estudio de la diversidad y las interacciones bióticas para la conservación y restauración de la vegetación de la Reserva de la Biósfera Ría Lagartos, Yucatán (segunda fase)”.

\section{AgRADECIMIENTOS}

A Ileana Lugo y Vanessa Hernández, estudiantes de la Universidad Autónoma de Yucatán, por su valiosa colaboración en el campo. Al personal de la Reserva de la Biósfera Ría Lagartos por las facilidades otorgadas.

\section{LITERATURA CITADA}

Arias-Medellín, L.A., C. Bonfil y T. Valverde. 2016. Demographic analysis of Agave angustifolia (Agavaceae) with an emphasis on ecological restoration. Botanical Sciences 94(3): 513-530. DOI: https://doi.org/10.17129/botsci.525

Arizaga, S. y E. Ezcurra. 2002. Propagation mechanisms in Agave macroacantha (Agavaceae), a tropical arid-land rosette. American Journal of Botany 89(4): 632-641. DOI: https://doi.org/10.3732/ajb.89.4.632

Badano, E. I. y F. I. Pugnaire. 2004. Invasion of Agave species (Agavaceae) in south-east Spain: invader demographic parameters and impacts on native species. Diversity and Distributions 10(5-6): 493-500. DOI: https://doi. org/10.1111/j.1366-9516.2004.00086.x

Bautista, F., O. Frausto, T. Ihl y Y. Aguilar. 2015. Actualización del mapa de suelos del estado de Yucatán, México: Enfoque geomorfopedológico y WRB. Ecosistemas y Recursos Agropecuarios 2(6): 303-315.

Carmona-Escalante, A., P. Guadarrama, J. Ramos-Zapata, S. Castillo-Agüero y N. M. Montaño. 2013. Arbuscular micorrhizal fungi associated with coastal vegetation in Chuburná, Yucatán, Mexico. Tropical and Subtropical Agroecosystems 16(3): 431-443.

Clark, P. J. y F. C. Evans. 1954. Distance to nearest neighbor as a measure of spatial relationships in populations. Ecology 35(4): 445-453. DOI: https://doi.org/10.2307/1931034

Colunga-García Marín, P., E. Estrada-Loera y F. MayPat. 1996. Patterns of morphological variation, diversity, and domestication of wild and cultivated populations of Agave in Yucatán, Mexico. American Journal of Botany 83(8): 1069-1082. DOI: https://doi. org/10.1002/j.1537-2197.1996.tb12805.x

Espejel, I., O. Jiménez-Orocio, G. Castillo-Campos, P. P. Garcillán, L. Álvarez, S. Castillo-Argüero, R. Durán, M. Ferrer, D. Infante-Mata, S. Iriarte, J. L. León de la Luz, H. López-Rosas, A. Medel Narváez, R. Monroy, P. MorenoCasasola, J. P. Rebman, N. Rodríguez-Revelo, J. SánchezEscalante y S. Vanderplank. 2017. Flora en playas y dunas costeras de México. Acta Botanica Mexicana 121: 39-81. DOI: https://dx.doi.org/10.21829/abm121.2017.1290

Flores, S. J. e I. Espejel. 1994. Tipos de vegetación de la Península de Yucatán. Etnoflora Yucatanense 3: 1-35.

Franco, A. y P. S. Nobel. 1988. Interactions between seedlings of Agave deserti and the Nurse Plant Hilaria rigida. Ecology 69(6): 1731-1740. DOI: https://doi.org/10.2307/1941151

García-Mendoza, A., P. Colunga-García Marín y R. Jr. Bye. 1993. Los usos de Agave angustifolia (Haw.), ancestro silvestre del henequén en su área de distribución geográfica. In: Peniche, R. y B. Santamaría (eds.). Memorias de la Conferencia Nacional sobre el Henequén y la Zona Henequenera de Yucatán. Gobierno del Estado de Yucatán, Consejo Nacional de Ciencia y Tecnología (CONACYT), 
Universidad Autónoma de Yucatán, Sostenibilidad Maya, Instituto Nacional de Investigaciones Forestales y Agropecuarias. 25-28 octubre 1992. Mérida, Yucatán, México. Pp. 92-112.

García-Moya, E., A. Romero-Manzanares y P. S. Nobel. 2011. Highlights for Agave productivity. Global Change Biology, Bioenergy 3(1): 4-14. DOI: https://doi. org/10.1111/j.1757-1707.2010.01078.x

González-Medrano, F. 2004. Las comunidades vegetales de México. Instituto Nacional de Ecología, Secretaría de Medio Ambiente y Recursos Naturales. México, D.F., México. 82 pp.

Hernández-Cerda, M. E. y G. Valdez-Madero. 2004. Sequía meteorológica. In: Martínez, J. y A. Fernández (comps.). Cambio climático, una visión desde México. Instituto Nacional de Ecología, Secretaría de Medio Ambiente y Recursos Naturales. México, D.F., México. Pp. 315-326.

Jordan, P. W. y P. S. Nobel. 1979. Infrequent establishment of seedlings of Agave deserti (Agavaceae) in the northwestern Sonoran Desert. American Journal of Botany 66(9): 10791084. DOI: https://doi.org/10.2307/2442574

Krebs, C. J. 1998. Ecological methodology. Wiley and Sons. New York, USA. 620 pp.

Krebs, C. J. 2009. Ecology, the experimental analysis of distribution and abundance. Pearson. New York, USA. $655 \mathrm{pp}$.

Leirana-Alcocer, J. y F. Bautista. 2014. Patrones de asociación entre la cobertura vegetal y la calidad del suelo en el matorral costero de la Reserva Ría Lagartos, Yucatán. Ciencia UAT 8(2): 44-53. DOI: https://doi.org/10.29059/ cienciauat.v8i2.297

León-Arteta, R. 2003. Manual edafológico de campo. 2a. ed. Textos Universitarios, Universidad Veracruzana. Xalapa, Veracruz, México. 205 pp.

Medina-van Berkum, P., V. P. Parra-Tablay J. L. Leirana-Alcocer. 2016. Recursos florales y colibríes durante la época seca en la Reserva de la Biosfera Ría Lagartos, Yucatán, México. Huitzil, Revista Mexicana de Ornitología 17(2): 244-250. DOI: https://doi.org/10.28947/hrmo.2016.17.2.254

Nobel, P. S. 1988. Environmental biology of agaves and cacti. Cambridge University Press. Cambridge, UK. 284 pp.
Oborny, B. 1994. Spacer length in clonal plants and the efficiency of resource capture in heterogeneous environments: A Monte Carlo simulation. Folia Geobotanica et Phytotaxonomica 29(2): 139-158. DOI: https://doi.org/10.1007/bf02803791

Owen, N. A., K. F. Fahy y H. Griffiths. 2015. Crassulacean acid metabolism (CAM) offers sustainable bioenergy production and resilience to climate change. Global Change Biology, Bioenergy 8(4): 737-7449. DOI: https:// doi.org/10.1111/gcbb.12272

Ramírez-Tobías, H. M., C. B. Peña-Valdivia y R. J. R. Aguirre. 2014. Respuestas bioquímico-fisiológicas de especies de Agave a la restricción de humedad. Botanical Sciences 92(1): 131-139. DOI: https://dx.doi.org/10.17129/botsci.156

Rocha, M., S. Good-Ávila, F. Molina-Freaner, H. T. Arita, M. Castillo, A. García-Mendoza, A. Silva-Montellano, B. S. Gaut, V. Souza y L. E. Eguiarte. 2006. Pollination biology and adaptive radiation of Agavaceae, with special emphasis on the genus Agave. Aliso 22(1): 329-344. DOI: https://doi.org/10.5642/aliso.20062201.27

Salazar-Solano, V. y A. Mungaray-Lagarda. 2009. La industria informal del mezcal bacanora. Estudios Sociales 17(33): 163-198.

StatPoint Inc. 2005. Statgraphics Centurion XV software Version 15.0.04. Herndon, USA.

Vázquez-Yañes, C., A. Orozco-Segovia, E. Rincón, M. E. Sánchez-Coronado, P. Huante, J. R. Toledo y V. L. Barradas. 1990. Light beneath the litter in a tropical forest: effects on seed germination. Ecology 71(5): 19521958. DOI: https://doi.org/10.2307/1937603

Winter, K., M. García y J. A. M. Holtum. 2014. Nocturnal versus diurnal $\mathrm{CO}_{2}$ uptake: how flexible is Agave angustifolia? Journal of Experimental Botany 65(13): 3695-3703. DOI: https://doi.org/10.1093/jxb/eru097

Zepeda, V., J. Golubov y M. C. Mandujano. 2017. Distribución espacial, estructura de tamaños y reproducción de Astrophytum ornatum (Cactaceae). Acta Botanica Mexicana 119: 35-49. DOI: http://dx.doi.org/10.21829/ abm119.2017.1230 